\title{
Monedas complementarias: dinero con valores
}

\author{
August Corrons \\ Universitat Oberta de Catalunya \\ acorrons@uoc.edu
}

Resumen: En este artículo se presentan y analizan las monedas complementarias: concepción, características, tipologías, situación actual, evolución e influencia sobre el sistema monetario.

Desde enfoques teóricos, a casos reales e incluso estudios científicos, todos los trabajos referenciados en este artículo analizan y demuestran las bondades de las monedas complementarias, así como su capacidad de influencia en la sostenibilidad de la vida en el planeta Tierra, tanto a nivel social como económico y ambiental.

Palabras clave: monedas complementarias; sostenibilidad; sistema monetario.

Abstract: This paper analyzes complementary currencies, their definition, characteristics, typology, current situation, evolution and their influence on the global monetary system.

From theoretical approaches, real cases and scientific studies, there are different references highlighting the benefits of complementary currencies and their influence on sustainability at different levels, social, economic and environmental.

Keyword: complementary currencies, sustainability, monetary system. 


\section{Introducción a las monedas complementarias}

\section{El concepto de complementariedad}

El concepto de monedas complementarias apareció por primera vez en un informe que Bernard Lietaer presentó a la Comisión Europea (Rizzo, 2003). Las monedas complementarias (también llamadas alternativas, comunitarias, locales/regionales o sociales), son sistemas monetarios que se crean al margen de las monedas oficiales del país y que tienen como objetivo fundamental la promoción de proyectos económicos, sociales y ambientales de carácter regional, al mismo tiempo que ponen en valor los activos y recursos locales que no se encuentran dentro de los círculos o circuitos ordinarios de intercambio debido a la escasez de la moneda oficial. Lietaer (2005) destaca que, si las monedas convencionales promueven una serie de valores, tales como autoridad central, jerarquía, competencia, causa-efecto, reduccionismo y tecnología, se necesitan monedas complementarias con otra serie de valores, tales como confianza mutua, igualdad, cooperación, sincronicidad, holismo y habilidades interpersonales.

Según Lietaer (2005), Lopezllera (2002) y Primavera (2001), el significado de los distintos calificativos con los que se llama a este tipo de monedas es el siguiente:

- Complementarias: porque se complementan con el dinero convencional, al que en ningún momento pretenden reemplazar.

- Alternativas: porque funcionan en lugar del dinero convencional en ciertos contextos.

- Comunitarias: porque funcionan en comunidades de personas o entidades que se relacionan directamente y desean establecer un medio de intercambio distinto.

- Locales/regionales: porque circulan en lugares y espacios delimitados.

- Sociales: porque son creadas, emitidas y controladas por grupos sociales.

En este artículo se ha optado por el uso del calificativo complementarias por ser el que mejor refleja una de las principales características de este tipo de monedas, la complementariedad. Consideramos que el resto de adjetivos (alternativas, comunitarias, locales/regionales o sociales) quedan implícitos, en mayor o menor grado, en el concepto general de monedas complementarias, puesto que, tal y como veremos más adelante, estas también promueven lo alternativo, lo comunitario, lo local/regional y lo social. 


\section{Evolución histórica y experiencias}

El dinero es definido como medio de intercambio, depósito de valor y unidad de cuenta. Sin embargo, solo en el actual período de la historia estas funciones residen en una única moneda oficial de escala nacional. A lo largo de la mayor parte de la historia, diferentes formas de dinero han cumplido estas funciones por separado, y se mantuvieron vigentes por siglos (Lietaer, 2005; Greco, 2001; Douthwaite, 1996).

Desde un punto de vista genérico, podemos remontarnos a los primeros sistemas de trueque de la humanidad para encontrar la sistemática de los sistemas monetarios sociales (Lietaer, 2005). Desde hace miles de años, distintas comunidades humanas han creado sus propias monedas con objeto de satisfacer las necesidades de sus miembros y resguardarse así de la inestabilidad económica. Desde entonces, $y$ hasta nuestros días, las monedas sociales han pasado por numerosos avatares, y en la actualidad se encuentran en muchísimos países del mundo. Las monedas nacionales, así como las supranacionales, han sido un claro obstáculo para su desarrollo, si bien algunos de los sistemas monetarios complementarios se basan en monedas que precisan de la redención última en moneda oficial.

En muchas comunidades, a lo largo de la historia, se han creado monedas locales (Lietaer, 2005). En el antiguo Egipto se crearon este tipo de monedas, y su existencia está íntimamente relacionada con su prosperidad económica. También en la Edad Media y en el sistema feudal se crearon este tipo de sistemas monetarios. Un ejemplo emblemático de monedas comunitarias se circunscribe al hecho de que las grandes catedrales de la Europa del Medioevo fueron financiadas con los denominados «breakteats» o monedas locales, y los trabajadores fueron remunerados con dichas monedas.

El primer modelo de intercambio es el denominado mercancía-mercancía (Lietaer, 2005). Con la aparición del dinero aparece el modelo mercancía-dinero-mercancía, facilitando los intercambios y la división del trabajo. El paso siguiente en la modelización de los intercambios fue el modelo dinero-dinero. Esta situación dio paso en nuestros días a la eclosión de la economía financiera que, según los defensores de la economía solidaria, se encuentra muy alejada de la economía real. De hecho, la economía financiera tiene su propia lógica al margen de la economía productiva.

Hay diversas experiencias documentadas del uso de monedas complementarias, que se combinan con dinero convencional, desde los años 30 del siglo xx. Se parte del uso de monedas complementarias en ese tiempo: en Alemania surgió el Wära, en Austria el Wörgl, y otras en los países escandinavos, Bulgaria, Canadá, Dinamarca, Ecuador, España, Francia, Italia, México, Países Bajos, Rumania, Su- 
ecia, Suiza, Finlandia y China (Lietaer, 2005; Lopezllera, 2002). Estas monedas respondieron a la necesidad de tener formas alternativas para comprar bienes para el abastecimiento familiar durante la depresión de 1929, si bien la mayoría de ellas fueron prohibidas por los gobiernos de los países después de un tiempo en funcionamiento. Tan solo una de ellas sobrevive hasta el día de hoy. Es el caso del Wir de Suiza.

Después de medio siglo, en 1983, Michael Linton ideó los LETS (Local Employment and Trade System), que surgieron en Canadá y que actualmente son el sistema monetario complementario más difundido en todo el mundo. Pronto se extendió a Inglaterra, Nueva Zelanda y Austria, pasando posteriormente a Estados Unidos, Australia y Europa (Lietaer, 2005). De forma independiente apareció en Francia el SEL (Système de Échange Local), en Japón el Fureai Ticket y en Estados Unidos otros sistemas que se han vuelto muy populares como son los Time Dollars y los Ithaca Hours.

Tal y como apunta Lopezllera (2002), el binomio dinero local-dinero convencional funciona con éxito en países económicamente poderosos, al igual que en países de economía dependiente como Brasil, Tailandia, Indonesia y Senegal.

Por todo ello, no ha de extrañar que en nuestro tiempo surjan nuevas formas de dinero. Tal y como apunta Weatherford (1998), estamos entrando en un período de transición en el que competirán múltiples sistemas generadores de dinero, sin que ninguno de ellos domine sobre el resto. Las monedas nacionales están perdiendo la importancia que tenían. Nos hallamos en los albores de la era del dinero.

Muchas monedas complementarias han aparecido durante momentos de crisis económicas en que escasea el trabajo remunerado y el dinero. Tal es el caso de Argentina, cuyo colapso económico sufrido en 2001, impulsó que se sumaran nuevos sistemas de crédito alternativos, o monedas complementarias, a los que ya existían y, en poco tiempo, llegaron a involucrar a cientos de miles de usuarios, fenómeno que se expandió hacia otros países de la región (Primavera, 2003).

En el contexto de crisis económica, no solo la gente de escasos recursos explora alternativas, también la gran empresa capitalista echa mano de distintas formas de atraer y conservar su clientela (Santana, 2008). Las llamadas millas que se otorgan a cambio de haber comprado billetes previamente en una línea aérea, si bien no son en rigor dinero, atraen cada vez más viajeros frecuentes. Hasta el punto que hoy en día las millas también sirven para pagar hoteles y restaurantes, de modo que se empiezan a tratar ya como monedas complementarias, aunque la gente no lo perciba de este modo. La idea se ha extendido a muy diversos servicios (regalos a los clientes frecuentes). Así mismo, los grandes almacenes lanzaron 
las tarjetas de puntos o monederos electrónicos para pagar parte de la siguiente compra en la misma cadena de almacenes.

El dinero alternativo de empresas capitalistas se asemeja al dinero complementario en el sentido de que se utiliza algo distinto al dinero convencional para realizar compras, ya sean puntos electrónicos, vales... No obstante, vale la pena reflejar una diferencia muy clara e importante a nivel conceptual: el primero fue ideado como parte de la competencia capitalista, para atar al cliente, para que regrese al mismo lugar donde ya compró, mientras que el segundo busca ofrecer al consumidor alternativas ante la carencia de dinero y la monopolización del mercado entre otras cosas (Santana, 2008).

\section{Características e impactos generados}

\section{Fundamentos}

Si bien las monedas complementarias siempre han existido de forma paralela a las monedas respaldadas por el Estado, en tiempos de recesión y crisis económica parecen ser más atractivas, lo que genera un nuevo ciclo de experimentación y crecimiento (Stodder, 1998), tal y como sucede con la actual crisis.

El precursor más lejano del dinero complementario podría ser el trueque, debido a que este facilita intercambios sin usar dinero convencional (Lietaer, 2005). Sin embargo, el trueque tiene la triple dificultad de que ambas partes quieran deshacerse de algo y que el objeto de su deseo lo tenga su contraparte, además de que sea equivalente en valor al que se posee. Por estas dificultades, desde los primeros tiempos, los pueblos comenzaron a usar como dinero cosas valiosas, o de uso frecuente, para establecer las equivalencias y realizar los intercambios. El dinero complementario es equivalente a usar algo reconocido por todos los miembros de un grupo como forma de pago. Funciona como facilitador del multitrueque, es decir, trueque no bilateral, sino multilateral o en múltiples direcciones. Las condiciones para pertenecer al grupo (ser reconocido como parte de este) son: llevar algo para intercambiar, aceptar la moneda complementaria en los pagos dentro del mismo grupo y comprar lo que otros llevan.

Las monedas complementarias constituyen un símbolo de valor que un grupo de personas acuerdan emitir y utilizar para realizar intercambios de bienes y servicios. Pero esta no es una definición muy exacta, porque el dinero oficial también puede ser definido de la misma manera, es decir, todo dinero es un acuerdo para utilizar algo como un símbolo de valor. La diferencia es que el dinero oficial o convencional es emitido por el Banco Central de cada país, bajo las órdenes del gobierno de ese país, mientras que las monedas complementarias son emitidas por la gente (Lietaer, 2005). 
Este tipo de monedas han surgido de la sociedad civil y el tercer sector (Douthwaite, 1996 y Greco, 2001), como parte de un movimiento de abajo hacia arriba que promueve innovaciones de base para apoyar un enfoque más radical con el desarrollo sostenible.

Tal y como apunta Lietaer (2005), las monedas nacionales convencionales y los sistemas monetarios han sido programados para generar competencia y mantener la escasez. Si existen otras monedas disponibles, tendrá sentido continuar usando las monedas convencionales para los negocios, adquirir un auto, pagar el combustible o la cuenta del teléfono; pero para comunicarse con los vecinos, ocuparse de las personas mayores o ampliar los horizontes educativos de los niños, tal vez deba contemplarse el uso de una moneda que favorezca la cooperación.

Las monedas complementarias, en general, no tratan de replicar todas las funciones del dinero convencional, sino que usualmente tienen un propósito especial, ya sea para proveer liquidez adicional cuando el medio de intercambio oficial es escaso, almacenando valor con ciertos propósitos o incluso incentivando ciertos tipos de comportamientos (Brenes, 2013).

Según Seyfang y Longhurst (2012), los sistemas monetarios complementarios están diseñados para promover el desarrollo sostenible mediante la localización del desarrollo económico, la creación de capital social, la sustitución del consumo de materiales, la valoración del trabajo que está marginado en los mercados de trabajo convencional, así como el desafío del sistema monetario basado en el crecimiento.

\section{Impacto social}

Para algunos autores, el principal objetivo de las monedas complementarias es mejorar el bienestar social de las comunidades donde circulan, puesto que permiten suplir necesidades psicológicas como el reconocimiento, el sentido de pertenencia y la autoestima a través de la interacción social en lugar del individualismo y el consumismo material (Ryan-Collins y otros, 2008). Esto puede ocurrir por varias vías, como por ejemplo con actos de solidaridad entre vecinos que promueve un sentido de comunidad y una construcción de confianza entre participantes (Cahn, 2000; Collom, 2008). Iniciativas de este tipo son especialmente útiles en áreas donde las comunidades se han fragmentado o existe poca confianza entre diferentes grupos sociales, permitiendo pues la participación de grupos excluidos (Seyfang y Smith, 2002).

Tal y como apunta Gómez (2008), todo el mundo tiene algo que ofrecer, incluyendo aquellos cuyas habilidades no se valoran en el mercado de trabajo formal, empoderando pues a grupos socialmente excluidos, e impulsando así la au- 
toestima, la confianza, la participación social y el bienestar. Según Ryan-Collins y otros (2008), todos estos aspectos, incluso en las pequeñas interacciones que se derivan de las transacciones con una motivación más económica, se suman al crecimiento del espíritu comunitario y las redes de amistad y colaboración.

\section{Impacto económico}

Las monedas complementarias son citadas por muchas organizaciones como un instrumento para construir circuitos locales de valor económico que evitan que la riqueza fluya fuera de la comunidad, para realizar un efecto multiplicador en la economía local y para promover la localización de los procesos (Hopkins, 2008; Douthwaite, 1996).

Así mismo, el trabajo informal, el intercambio de habilidades, el voluntariado y el trabajo doméstico, todos ellos cruciales para la economía de mercado, pueden ser efectivamente valorados, reconocidos, recompensados e incluso intercambiados gracias a las monedas complementarias. Esto puede ayudar a contrarrestar la explotación de la mano de obra a través del empleo formal y ayudar a construir relaciones económicas donde se valore la cooperación y el intercambio.

Añadir también que este tipo de monedas ofrecen un medio complementario de acceso a bienes y servicios a personas financieramente excluidas o que no pueden encontrar empleo formal (Williams y otros, 2001). Del mismo modo, se argumenta que las monedas complementarias pueden apoyar el desarrollo económico sostenible a través de las pequeñas empresas locales que demuestran mayor lealtad a las comunidades locales, mediante sistemas de crédito mutuo entre empresas, lo que permite comerciar entre sí sin necesidad de dinero nacional. Algunas de estas monedas incluso apoyan específicamente a las empresas sociales y a los negocios con enfoque sostenible.

\section{Impacto ambiental}

Las monedas complementarias tienen impactos ambientales positivos, puesto que reducen la huella ecológica a través de la creación de modelos de consumo más locales y la sustitución de las importaciones, reduciendo así la energía necesaria para el transporte (Douthwaite, 1996). Así mismo, facilitan el intercambio de recursos y la disponibilidad de un mercado para la venta de productos producidos localmente con recursos locales e incluso la reutilización de productos.

Algunas monedas complementarias estimulan un comportamiento más pro-ambiente, como por ejemplo, incentivando a los ciudadanos a participar en programas de reciclaje o estimulando la compra de productos más sostenibles y/o utilizando el transporte público. Así mismo, las monedas complementarias 
pueden fomentar el desarrollo de nuevas tecnologías más ambientales, como por ejemplo, en el caso de las energías renovables, aumentando la inversión de capital mediante la emisión de notas respaldadas por la producción de energía.

\section{Tipologías}

\section{Clasificación de Seyfang y Longhurst}

En el presente artículo se utiliza la clasificación de monedas complementarias propuesta por Gill Seyfang y Noel Longhurst en 2013. Se trata de una clasificación que analiza a las monedas complementarias en las tres esferas de la vida (ambiental, social y económica), lo cual facilita un análisis adecuado de dichas dimensiones y su influencia sobre la sostenibilidad global del sistema monetario.

Hay otra serie de clasificaciones que no serán presentadas, no por su falta de rigurosidad o fiabilidad, sino porque se alejan del objetivo perseguido con la presentación de la clasificación anterior. Tal sería el caso de las propuestas por Jérôme Blanc en 2011, Margrit Kennedy y Bernard Lietaer en 2004, Siglinde Bode en 2004 o Jens Martignoni en 2012.

\section{Presentación de las distintas tipologías}

La clasificación propuesta por Gill Seyfang y Noel Longhurst en 2013, presenta las monedas complementarias como intervenciones para fortalecer la solidaridad local, ofrecer liquidez adicional e incentivar las motivaciones ambientales, empleando a las monedas en la línea de establecer el triple balance de la esfera social, ambiental y económica.

Se clasifica a las monedas complementarias en cuatro categorías distintas, cuya presentación conceptual es la siguiente:

- Service credit (SC o créditos de servicios). Son las monedas basadas en el tiempo, el cual se gana ayudando a otra persona u organización. El tiempo de todos vale lo mismo, independientemente del servicio prestado. Los SC se pueden gastar en los servicios ofrecidos por otros miembros. Estos sistemas son a menudo conocidos como bancos de tiempo. Algunos se organizan en un barrio o en una comunidad, siempre a cargo de voluntarios, si bien algunos están impulsados por ciertas instituciones. Son sistemas que a menudo se centran en un sector específico, como son la salud, la educación o la justicia penal.

- Mutual exchange (ME o intercambios mutuos). Son las monedas que son creadas por sus propios miembros. Estos miembros anuncian sus 
ofertas y sus demandas en un directorio, mientras que un sistema de contabilidad central se encarga de registrar las transacciones. La moneda propiamente dicha se crea cuando se realiza una transacción de compra/ venta en la que, tanto el comprador como el vendedor, se comprometen con el sistema. Los LETS (Local Exchange and Trading Systems) son una de las formas más conocidas de sistema de intercambio mutuo. Los ME tienden a existir en el contexto de la sociedad civil, a menudo con poco apoyo por parte del Estado u otras fuentes de financiación.

- Local currencies (LC o monedas locales). Son monedas que están geográficamente delimitadas (a menudo en forma de billetes o notas de papel), que circulan dentro de una determinada región. Tienen como objetivo la promoción de la actividad económica en la región y el apoyo a la economía local, previniendo que el dinero escape de la localidad. Existen varios modelos diferentes de LC, como por ejemplo el modelo de las Ithaca Hours (EE.UU.), el Regiogeld (Alemania), el Banco Palmas (Brasil) y el Transition Currencies (Reino Unido). Si bien todos estos tienen una moneda física, en papel, hay otros casos en los que se está experimentando con plataformas electrónicas, incluyendo tarjetas de débito y transacciones a través de teléfonos móviles.

- Barter markets (BM o mercados de trueque). Son monedas que están destinadas a superar la escasez de dinero en efectivo y a facilitar el intercambio entre un grupo de usuarios, por lo general en un mercado regular. $\mathrm{Al}$ igual que las $\mathrm{LC}$ anteriormente mencionadas, los BM tienden a utilizar los registros y apuntes físicos para controlar los saldos, que a menudo se emiten a los nuevos usuarios (como un préstamo sin intereses) para que puedan participar en el mercado. Los mercados de trueque se asocian a menudo con la idea de prosumidores (individuos que producen y consumen al mismo tiempo). El ejemplo más conocido corresponde a los mercados de trueque de Argentina, que crecieron de tamaño significativamente en la década de 2000, en parte como respuesta a los problemas económicos presentes en el país. 


\section{Situación actual}

\section{Consideraciones previas}

A continuación se presenta un análisis del estado actual de las monedas complementarias en el mundo, en base a los resultados del único estudio existente a nivel internacional: el de Seyfang y Longhurst (2013). De cara a poder interpretar estos resultados, hay que remarcar que se trata de un estudio en el que se han contabilizado, para un determinado país, los sistemas monetarios complementarios que presentaban como mínimo 5 proyectos activos en 2013. Por tanto, las iniciativas emergentes o con menos de cinco proyectos activos en un mismo país no están incluidas en este análisis global, así como tampoco están incluidos los sistemas de intercambio comercial (monedas complementarias de índole meramente capitalista, anteriormente mencionadas), ni los programas de fidelización basados en incentivos para la compra de bienes y servicios sostenibles o el reciclaje.

\section{Identificación de monedas complementarias}

Según Seyfang y Longhurst (2013), existe un total de 38 grupos de monedas de base nacional, en 23 países de los seis continentes, lo que representa un total de 3.418 proyectos o iniciativas locales. Se entiende por grupo de monedas al conjunto de como mínimo 5 proyectos, de la misma índole, activos en 2013 en un mismo país. Cada uno de las iniciativas se cataloga en la Tabla 1 dentro de uno de los cuatro tipos principales de monedas complementarias anteriormente mencionados.

\begin{tabular}{|c|c|c|c|}
\hline & No proyectos & No países & No continentes \\
\hline Créditos de servicios & 1.715 & 11 & 4 \\
\hline Intercambios mutuos & 1.412 & 14 & 5 \\
\hline Monedas locales & 243 & 6 & 4 \\
\hline Mercados de trueque & 48 & 4 & 2 \\
\hline
\end{tabular}

Presencia de las distintas tipologías de monedas complementarias. Fuente: Corrons (2015), adaptado de Seyfang y Longhurst (2013).

A continuación se describe, para cada una de las cuatro tipologías de monedas complementarias, las características más importantes, así como su frecuencia temporal y su distribución geográfica. 


\section{Créditos de servicios (SC)}

El tipo más común de moneda complementaria que fue encontrado en el estudio de Seyfang y Longhurst (2013) fue el de créditos de servicios, con 1.715 proyectos $(50,2 \%$ del total), repartidos en 11 países y en 4 continentes.

Los SC, por lo general, apuntan a la construcción de capital social, la inclusión y la cohesión por el apoyo entre vecinos, la asistencia social, las actividades basadas en la comunidad, así como el trabajo a nivel de programas de voluntariado recíproco.

Para los SC es fundamental la unidad monetaria basada en el tiempo. Los participantes obtienen un crédito en tiempo por cada hora que destinan a ayudar a alguien, independientemente del servicio prestado. Estos créditos se pueden guardar para usarlos en un futuro, se pueden donar a otra persona, o se pueden usar para comprar servicios de otros miembros.

Tal y como apunta Cahn (2000), esto representa un rechazo radical a las valoraciones del mercado de trabajo, puesto que el tiempo de todos vale lo mismo.

El SC más conocido y popular corresponde a los bancos del tiempo, si bien no todos los proyectos de créditos de servicios identificados en el estudio de Seyfang y Longhurst (2013) son formalmente parte de la red internacional de bancos del tiempo.

Uno de los primeros ejemplos de banco del tiempo se registra en Japón a partir de 1973, el Fureai Kippu, si bien no puede considerarse como el propulsor de este tipo de sistemas. En Estados Unidos, Edgar Cahn fue quien desarrolló realmente la idea de bancos del tiempo, en 1986, para utilizar las habilidades y recursos sin explotar en los barrios desfavorecidos, para reconstruir las comunidades y para restablecer la dignidad de las personas excluidas socialmente. Este modelo se expandió rápidamente a través de los Estados Unidos, pasando posteriormente al Reino Unido en 1997, a través de David Boyle y la New Economics Foundation (NEF). Desde entonces, existen redes muy fuertes e importantes tanto en el Reino Unido y como en los Estados Unidos, las cuales han desarrollado nuevas metodologías y han mejorado las prácticas, al mismo tiempo que han apoyado a nuevos proyectos a nivel internacional, como por ejemplo en Italia, España, Portugal, Nueva Zelanda, Finlandia, Canadá y Japón.

\section{Intercambios mutuos (ME)}

Según el estudio de Seyfang y Longhurst (2013), la segunda categoría más importante de monedas complementarias corresponde al intercambio mutuo, representado por 1.412 proyectos (41,3\% del total), incluidos en 14 países y en 5 continentes. 
Los ME son creados durante el propio acto de compra o gasto: el crédito de una persona es igual al débito de otra, de modo que las cuentas siempre suman cero. Tanto el valor como la utilidad de la moneda se conservan gracias a la confianza entre los miembros para cumplir sus compromisos (o deudas).

Los intercambios mutuos suelen operar dentro de un área geográfica definida, proporcionando a los usuarios el acceso al crédito sin intereses, que puede ser gastado dentro del círculo de comercios inscritos en el programa. Los miembros anuncian sus ofertas o demandas en un directorio, de modo que cuando se realiza una transacción esta queda contabilizada en el propio sistema, ya sea a través de un talonario donde se apuntan los participantes y el importe de la transacción, o ya sea a nivel tecnológico. Algunos proyectos vinculan el valor de su moneda a la moneda nacional, otros prefieren un sistema basado en el tiempo, y algunos incluso mezclan el tiempo con los valores propios de la moneda.

Todo indica que, si bien los intercambios mutuos están claramente dirigidos a apoyar las economías locales, son los beneficios sociales y de fomento de la comunidad los que tienen el mayor impacto a través de las redes sociales que fomentan (Seyfang, 2001a, 2001b; Williams y otros, 2001).

El ejemplo más conocido son los LETS (Local Exchange and Trading Systems), que nacieron en la isla de Vancouver, Canadá, en 1983 por parte del activista comunitario Michael Linton. Su propósito inicial fue el de ser un dinero de emergencia durante la recesión. Los LETS, generalmente surgidos de la sociedad civil, se extendieron rápidamente a través de Canadá, Reino Unido, Nueva Zelanda y Australia durante los años 1980 y 1990, a través de redes de activistas verdes (Ekins, 1986).

El crecimiento de los LETS alcanzó su punto máximo a finales de 1990 en el Reino Unido; debiendo esperar unos años más para alcanzar su madurez a nivel del resto de Europa. Existen adaptaciones de los LETS en Francia, Hungría, Alemania, Austria, Suiza y Australia, y modelos similares que han surgido en África del Sur, Japón y Canadá. Otros países se han inspirado en los LETS, dando lugar a nuevas formas y sistemas híbridos. Así por ejemplo, el modelo CES (Community Exchange System) está inspirado en LETS y proporciona una plataforma electrónica en la que pueda tener lugar el intercambio mutuo. Este sistema se originó en Sudáfrica, pero se ha extendido a nivel internacional y ha sido utilizado por muchos de los sistemas de intercambio mutuo existentes.

\section{Monedas locales (LC)}

El tercer grupo de monedas complementarias según Seyfang y Longhurst (2013) está limitado geográficamente, puesto que las monedas están respaldadas en la 
mayoría de los casos por el dinero oficial que existe a nivel local. Comprende 243 proyectos (7,1\% del total), en 6 países diferentes de 4 continentes.

Las LC buscan circular exclusivamente dentro de una región geográficamente acotada, aumentando el multiplicador económico local y el apoyo a las empresas locales. En algunos casos se consiguen a través de la conversión con la moneda nacional, formando así vales de intercambio local que son canjeables solo dentro de ciertas áreas o con las empresas participantes. Una vez emitidos, circulan libremente hasta que se convierten de nuevo a moneda nacional.

Estas monedas tienen el propósito de complementar la moneda nacional, aumentar la velocidad de los intercambios locales, pero sin suplantar la moneda nacional o el comercio interregional. Se presta especial atención a las características de seguridad; hasta el punto que existen muchos sistemas que utilizan impresoras de divisas convencionales para producir sus billetes, es decir, a prueba de falsificaciones.

La categoría de las LC incluye las Horas, que se originaron en Ithaca, Estado de Nueva York, en 1991. Existen también otros proyectos dentro los Estados Unidos, en Canadá y en otros países, la mayoría de ellos ligados a grupos ecologistas y alternativos, que utilizan la moneda local para impulsar la actividad económica local. El sistema alemán Regiogeld (dinero regional) es un proyecto que se centra más en el desarrollo económico local y que tiene una fuerte red de intercambio. Los bancos comunitarios de Brasil, como parte de un movimiento basado en la economía solidaria hacia el desarrollo económico y el empoderamiento ciudadano, tienen como objetivo impulsar la actividad económica local en regiones marginales.

Un modelo de LC llegó al Reino Unido en 2007 y ha estado creciendo en forma de cinco monedas distintas, conocidas como Transition Currencies. Aunque no hay ninguna actividad de red formal entre ellas, se comparte el aprendizaje y la experimentación con los mecanismos de pago electrónico para aumentar la captación de usuarios. Estas LC del Reino Unido están todas asociadas, en mayor o menor grado, con el decrecimiento de las ciudades en transición, y tienen por objeto aumentar la capacidad de recuperación económica local (Graugaard, 2012; Ryan-Collins, 2011).

\section{Mercados de trueque (BM)}

La cuarta categoría, correspondiente a los mercados de trueque, es representada según Seyfang y Longhurst (2013) por 48 proyectos (1,4\% del total), que operan en 4 países de 2 continentes. 
Los $\mathrm{BM}$ son un híbrido entre las $\mathrm{LC}$ y los $\mathrm{ME}$, comprendiendo una nueva infraestructura para que las personas puedan intercambiar bienes y servicios dentro de un evento específico, en un sitio limitado, sin la necesidad de moneda corriente. Los usuarios de los BM se unen a un club local en el que consiguen la moneda local (préstamos sin intereses), para consumir dentro de los propios mercados. Se trata de monedas que posteriormente no son convertibles a la moneda oficial (Pearson, 2003).

Los BM surgieron por primera vez en Bernal, Buenos Aires, como una iniciativa de sostenibilidad instigada por una ONG ambiental en 1995. Emergieron en un contexto de desindustrialización y crisis fiscal, se expandieron rápidamente durante el colapso financiero argentino de 1999 a 2002, y se convirtieron en una forma de vida para un grupo demográfico muy amplio. No obstante, los clubes de trueque argentinos fueron castigados por la rivalidad entre redes y sufrieron un colapso catastrófico en credibilidad en 2002 (North, 2007). Sin embargo, algunos sistemas argentinos aún permanecen y modelos similares han sido adoptados en Venezuela y México, donde se han mantenido estrechamente asociados a las ideas de la economía solidaria. Los mercados de trueque informales son también operativos en otros países de América del Sur.

Ha surgido también en Quebec, Canadá, una región con un fuerte movimiento de economía social. Sin embargo, en Canadá, este sistema tiene un mayor énfasis en el apoyo al desarrollo sostenible a través de la reutilización de los bienes, que la ideología de solidaridad económica que motiva a los sistemas de México y Sudamérica.

\section{Distribución espacial y temporal}

Habiendo identificado ya los cuatro tipos principales de monedas complementarias, su prevalencia, así como sus características y objetivos particulares, la atención se centra ahora en la difusión geográfica de estas iniciativas. En la Tabla 2 y Figura 1 se presentan datos sobre el número de proyectos de monedas complementarias, mostrando cómo se distribuyen los cuatro tipos a través de los diferentes continentes. 


\begin{tabular}{|c|c|c|c|c|c|}
\hline & Total (\%) & SC (\%) & ME (\%) & LC (\%) & BM (\%) \\
\hline Europa & 68,3 & 44,4 & 54,1 & 1,5 & $\ldots$ \\
\hline Asia & 16,6 & 68,7 & 7,9 & 23,4 & $\ldots$ \\
\hline América del Norte & 9,8 & 79,3 & 11,7 & 4,5 & 4,5 \\
\hline América del Sur & 2,7 & $\ldots$ & $\ldots$ & 64,5 & 35,5 \\
\hline Australia / Nueva Zelanda & 1,7 & 42,1 & 57,9 & $\ldots$ & $\ldots$ \\
\hline África & 0,9 & $\ldots$ & 100,0 & $\ldots$ & $\ldots$ \\
\hline
\end{tabular}

Distribución geográfica de las monedas complementarias, por región y tipo. Fuente: Corrons (2015), adaptado de Seyfang y Longhurst (2013).

El estudio de Seyfang y Longhurst (2013) revela que Europa tiene el mayor número de proyectos, con 2.333 iniciativas de un total de 3.418 (es decir, el $68,3 \%)$, de los cuales más de la mitad $(54,1 \%)$ son del tipo de intercambio mutuo $\mathrm{ME}$, el 44,4\% son créditos de servicios $\mathrm{SC}$ y únicamente el 1,5\% son monedas locales LC. A continuación sigue Asia con el 16,6\% de los proyectos de monedas complementarias, de los cuales más de dos tercios $(68,7 \%)$ son de programas de créditos de servicios SC, el 23,4\% son de monedas locales LC, siendo el resto de intercambios mutuos ME. América del Norte es la tercera región más poblada en cuanto a monedas complementarias, con un 9,8\% de los proyectos, de los cuales la gran mayoría $(79,3 \%)$ son los créditos de servicios SC (principalmente en los EE.UU). América del Sur representa el 2,7\% de los proyectos de monedas complementarias, siendo exclusivamente de monedas locales LC (64,5\%) y de mercados de trueque BM $(35,5 \%)$. Australia y Nueva Zelanda tienen tan sólo el $1,7 \%$ de las iniciativas del mundo, divididas entre los intercambios mutuos $\mathrm{ME}$ $(57,9 \%)$ y los créditos de servicios SC (42,1\%). Por último, los países de África tratan exclusivamente con intercambios mutuos $\mathrm{ME}$, constituyendo el 0,9\% del total a nivel internacional. 
Figura 1: Distribución geográfica de las monedas complementarias, por región y tipo.

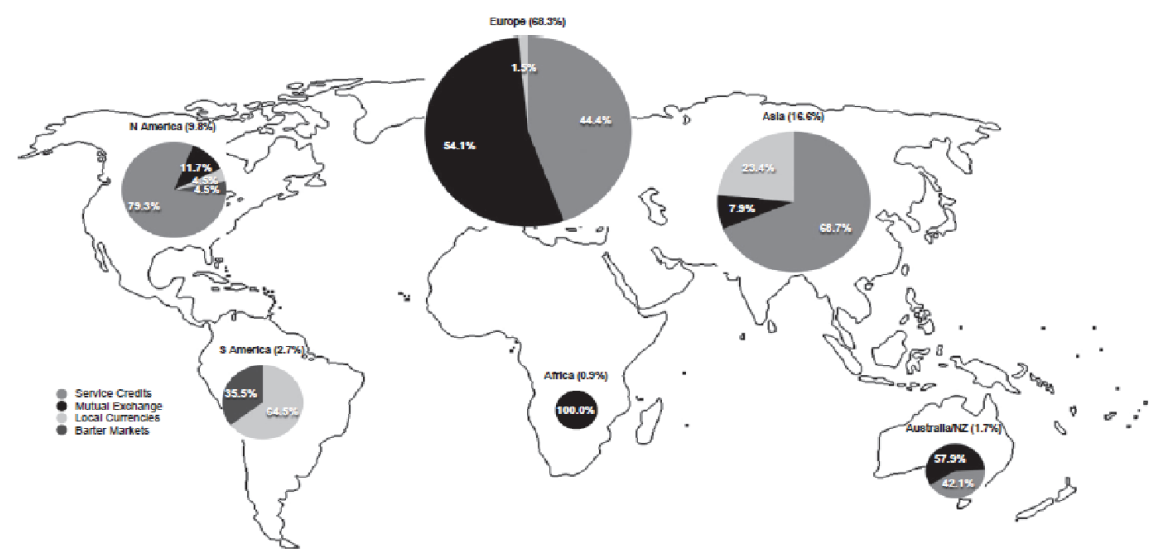

Fig. 1. Geographical distribution of community aurrendes, by region and type.

Fuente: Seyfang y Longhurst (2013).

Estos modelos de monedas complementarias han viajado de un país a otro a través de las últimas tres décadas aproximadamente, adaptando y evolucionando a lo largo del camino (Ekins, 1986). En la Figura 2 se muestra una línea de tiempo para cada uno de los cuatro modelos de monedas complementarias, mostrando cómo el modelo ha sido adoptado en el seno de diferentes países.

Figura 2: Evolución temporal de las monedas complementarias y difusión geográfica.
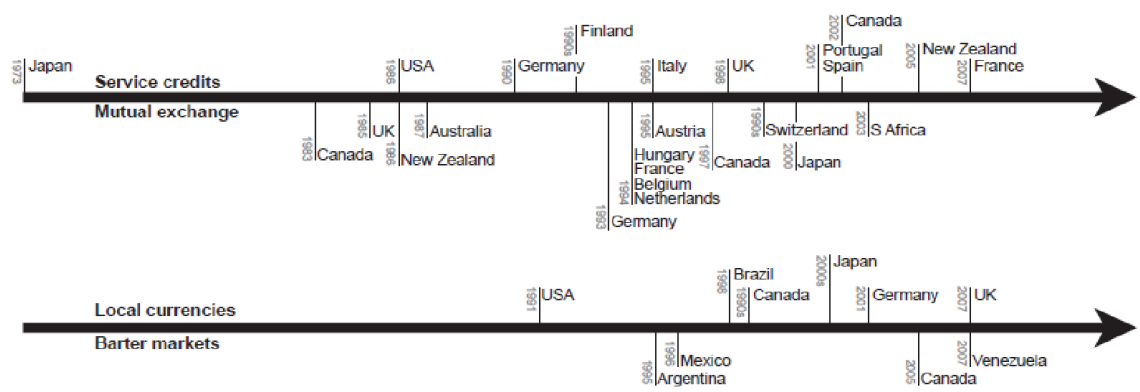

Fig. 2. Timelines showing geographical diffusion of community currencies.

Fuente: Seyfang y Longhurst (2013). 


\section{Sostenibilidad social, económica y ambiental}

Veamos cómo la caracterización tipológica de las monedas complementarias influye en sus grados de sostenibilidad. Es decir, el propio diseño de cada tipo de sistema monetario complementario afecta a la hora de fomentar más o menos lo ambiental, lo social o lo económico.

La presentación escogida en la Figura 3 para mostrar este análisis se realiza a través de un espacio triangular donde cada vértice representa a uno de los tres grados de sostenibilidad, y en el que se asignan las posiciones relativas de distintos tipos de monedas (Seyfang y Longhurst, 2013). Si bien este posicionamiento es una mera simplificación, revela aspectos y características muy interesantes.

Figura 3: Sostenibilidad de las distintas tipologías de monedas complementarias.

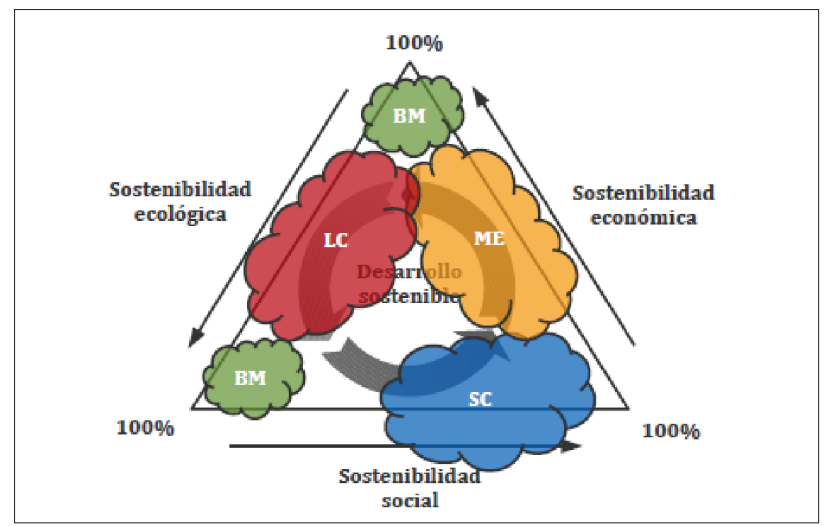

Fuente: Corrons (2015), adaptado de Seyfang y Longhurst (2012).

Seyfang y Longhurst (2013) demuestran que las monedas analizadas, dentro de cada una de las cuatro tipologías de monedas complementarias existentes, tienden a converger entre ellas. Independientemente del país de origen, existe congruencia de fines entre monedas de un mismo tipo. Por el contrario, las cuatro tipologías de monedas complementarias divergen entre sí, ocupando cada una de ellas distintas zonas del triángulo de representación. Así por ejemplo, las monedas de intercambio mutuo ME se encuentran ubicadas entre los objetivos económicos y sociales, mientras que las de créditos de servicios SC presentan mayoritariamente objetivos sociales, si bien también, en parte, ecológicos. En el caso de las monedas locales LC, se mueven entre metas económicas y ambientales. La única excepción corresponde a los mercados de trueque BM, donde hay una profunda diferencia entre el modelo económico del Trueque argentino y el modelo ambiental del Troc-tes-Trucs canadiense. 


\section{Sostenibilidad del sistema monetario}

\section{Introducción a los sistemas complejos}

De la evolución de la teoría general de los sistemas (TGS), el enfoque sistémico y el pensamiento complejo, surge el concepto de sistema complejo. La teoría de los sistemas complejos define a estos como los sistemas que no se pueden descomponer, aquellos cuyas partes no pueden ser aisladas ni modificadas independientemente unas de otras. Un sistema complejo está caracterizado por una densa red de interrelaciones y flujos de energía entre los elementos que lo conforman, los cuales, a su vez, pueden ser de naturaleza diversa.

Cuanto más complejo sea un sistema, mayor número de relaciones y mecanismos de feedback o retroalimentación existirán entre sus elementos (García, 2006). En un sistema complejo, las posibilidades de regulación son siempre mayores que en uno simple $y$, en consecuencia, la estabilidad frente a las fluctuaciones de los factores externos es también mayor. Todos los sistemas complejos cuentan con propiedades intrínsecas reguladoras o emergentes, que actúan para alcanzar un estado de equilibrio relativo en el sistema.

Los sistemas complejos, ya sean ecosistemas, organismos vivos o economías, se entienden como materia-energía y sistemas de flujo de información. Décadas de estudio de los ecosistemas naturales han llevado a una comprensión matemática acerca de cómo una estructura en red afecta a la viabilidad a largo plazo de un ecosistema, en función de la eficiencia (habilidad del sistema de procesar cantidades de materia relevante, flujos de energía o de información) y la resiliencia (habilidad del sistema de recobrarse de disturbios) (Ulanowicz y otros, 2008). Estos estudios demuestran que la naturaleza no actúa para alcanzar la máxima eficiencia, sino que lo hace para encontrar un punto óptimo de equilibrio entre la eficiencia y la resiliencia.

Según Ulanowicz y otros (2008), la resiliencia de un sistema complejo se potencia con la diversidad y con las interconexiones, dado que hay más canales de interacción a los que recurrir en tiempos de problemas o cambios. Por otro lado, la diversidad y las conexiones juegan un importante papel en la eficiencia, pero en el sentido opuesto: la eficiencia crece a través de la racionalización, proceso en el cual la diversidad y las conexiones decrecen.

Para un sistema en red determinado, el punto óptimo de equilibrio es único. La curva que relaciona la sostenibilidad con sus propiedades emergentes presenta cierta asimetría, de modo que para lograr el punto óptimo la resiliencia es el doble de importante que la eficiencia (Lietaer y otros, 2008). No obstante lo anterior, tal y como se muestra en la Figura 4, todos los ecosistemas presentan unas 
condiciones todavía sostenibles dentro de un rango muy estrecho y específico alrededor del punto óptimo, llamado ventana de viabilidad (Ulanowicz, 2008).

Todo lo anterior, independientemente de que haya sido analizado en ecosistemas naturales, viene desde la pura estructura de un sistema complejo y, por tanto, es válido para cualquier red compleja con una estructura similar, al margen de lo que se esté procesando en el sistema: biomasa en un ecosistema, información en un sistema biológico, o dinero en un sistema económico (Lietaer y otros, 2008).

Figura 4: Balance óptimo entre eficiencia y resiliencia para la sostenibilidad de un sistema. Ventana de viabilidad.

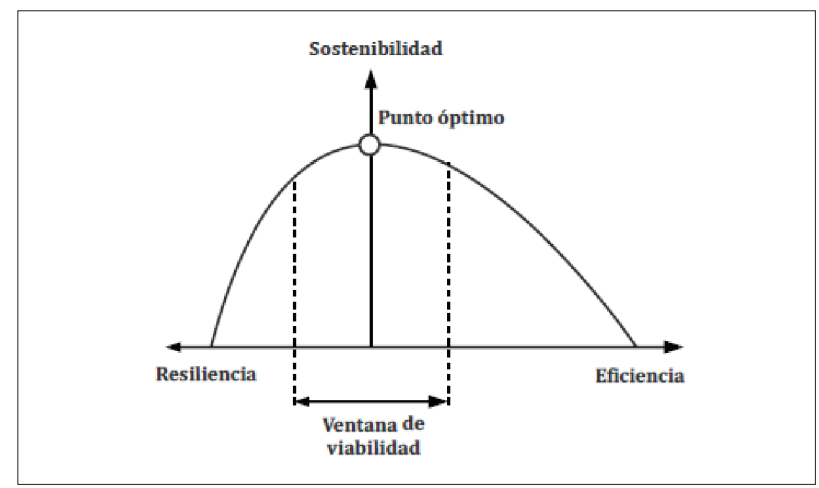

Fuente: Corrons (2015), adaptado de Lietaer y otros (2012).

La utilización conceptual de las redes de flujo complejas en el sistema monetario es precisamente el punto de partida para poder analizar la influencia de las monedas complementarias en la sostenibilidad de este sistema.

\section{Sistema monetario actual}

La situación del sistema monetario actual es tal que se está alejando cada vez más del punto óptimo, debido a su exclusivo énfasis en la eficiencia. Prevalece la creencia general de que todas las mejoras tienen que ir en esa misma dirección, la cual aleja al sistema todavía más de la sostenibilidad óptima (Lietaer y otros, 2008).

En la Figura 5 se refleja cómo el actual sistema monetario es excesivamente eficiente, en el sentido que es capaz de manejar elevadísimos volúmenes de información, mercancías... Diariamente se realizan intercambios monetarios equivalentes a unos 4 trillones de dólares sobre un PIB de 78 trillones de dólares. Sin embargo, un exceso de eficiencia bajo el esquema conceptual de una economía integrada en un ecosistema con recursos finitos, y sujeto a rendimientos decre- 
cientes, puede ser perjudicial. Ello justificaría en parte la recurrencia de las crisis, puesto que estamos situados en un punto muy eficiente pero emplazado más allá del umbral de la sostenibilidad (Lietaer y otros, 2008).

Figura 5: Posición del sistema monetario actual con relación a la zona de viabilidad.

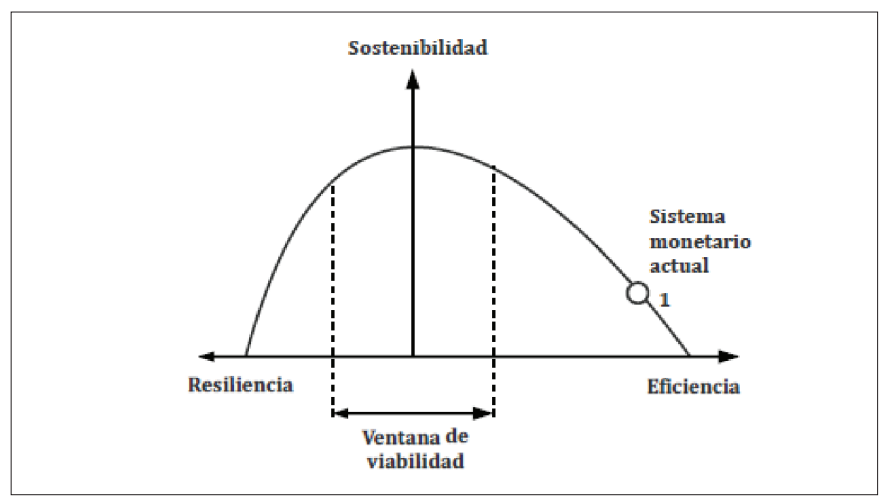

Fuente: Corrons (2015), adaptado de Lietaer y otros (2012).

La monocultura global del dinero como deuda bancaria se justifica técnicamente sobre la base de la eficiencia en la formación de los precios y el cambio dentro de cada país. Existe una regulación masiva para mantener esos monopolios, tal y como se refleja en la Figura 6. Internacionalmente, el cambio flotante se había justificado también porque es más eficiente. De forma análoga, en donde haya una crisis bancaria, al banco que es ya demasiado grande para caer invariablemente se le anima a absorber a los más pequeños, reduciendo más la diversidad institucional. Esto deja al sistema entero sin diversidad ni conexiones $y$, por tanto, con insuficiente capacidad de respuesta o resiliencia. Tal sistema es un accidente esperando ocurrir, condenado al colapso, por muy bien que se intente gestionar (Lietaer y otros, 2008). 
Figura 6: Efecto del monopolio del dinero como deuda bancaria.

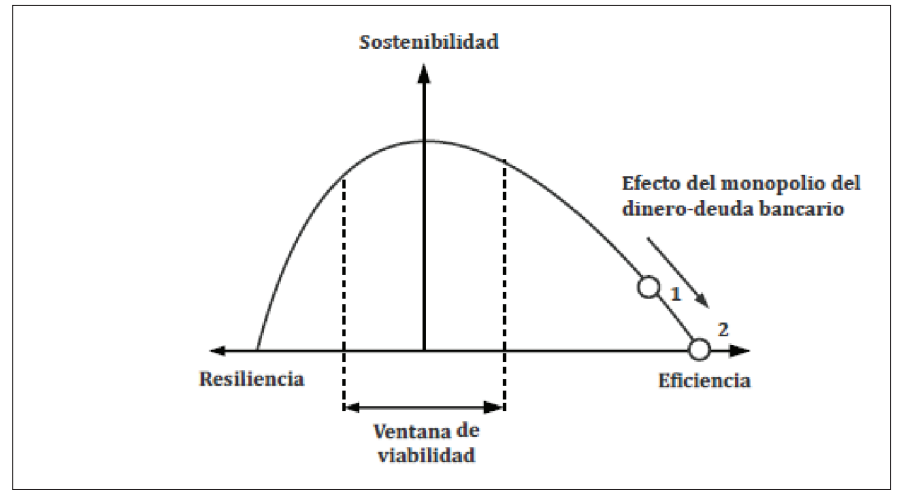

Fuente: Corrons (2015), adaptado de Lietaer y otros (2012).

Así mismo, cuando el sistema monetario colapsa, momentáneamente ve reducida de forma drástica su eficiencia, trasladándose pues a la zona de máxima resiliencia. Pero dicha situación es rápidamente intervenida por políticas o convenciones globales, empujando nuevamente al sistema hacia un punto muy cercano al previo a la crisis, es decir, a un punto excesivamente eficiente (Lietaer $y$ otros, 2008), tal y como se aprecia en la Figura 7.

Figura 7: Recuperación del sistema monetario tras un colapso.

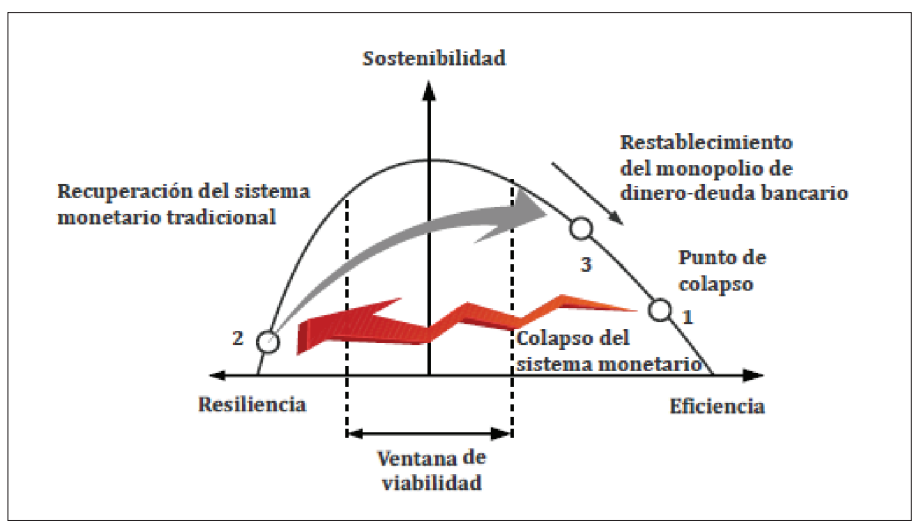

Fuente: Corrons (2015), adaptado de Lietaer y otros (2012).

Los ajustes realizados tras una crisis no se producen a través del aumento de la resiliencia sino de la eficiencia, devolviendo al sistema a un punto inestable. Todo lo contrario de lo que sucede en la naturaleza, donde tras un colapso o crisis el sistema recupera su eficiencia progresivamente, sin perder mucha resiliencia, y acercándose cada vez más a su punto óptimo de sostenibilidad. 


\section{Sistema monetario complementario}

La idea económica convencional asume de facto el monopolio de las monedas nacionales como un hecho incuestionable. En contraste, hay evidencias que apuntan a la conclusión de que la sostenibilidad monetaria requiere de una diversidad de sistemas de moneda, de manera que puedan emerger múltiples y diversas vías de cambio y conexión monetaria (Lietaer y otros, 2008).

Esto incuestionablemente reduce la eficiencia, pero también incrementa la capacidad de respuesta de la economía en general al facilitar las transacciones que de otro modo no ocurrirían, a través de una diversidad y unas conexiones que de otro modo no existirían (Lietaer y otros, 2008).

El efecto de las monedas complementarias puede tener lugar en cualquier momento, ya sea antes o después de un colapso o crisis propiamente dicha. En el primer caso se trata de anticiparse, en el segundo caso se trata de reaccionar. Si sucede antes del colapso, la Figura 8 muestra cómo su efecto se traducirá en un acercamiento progresivo hacia la zona de sostenibilidad viable, en base a un aumento de la resiliencia del sistema y, por tanto, a una pérdida de su eficiencia (Lietaer $y$ otros, 2008).

Figura 8: Efecto de las monedas complementarias.

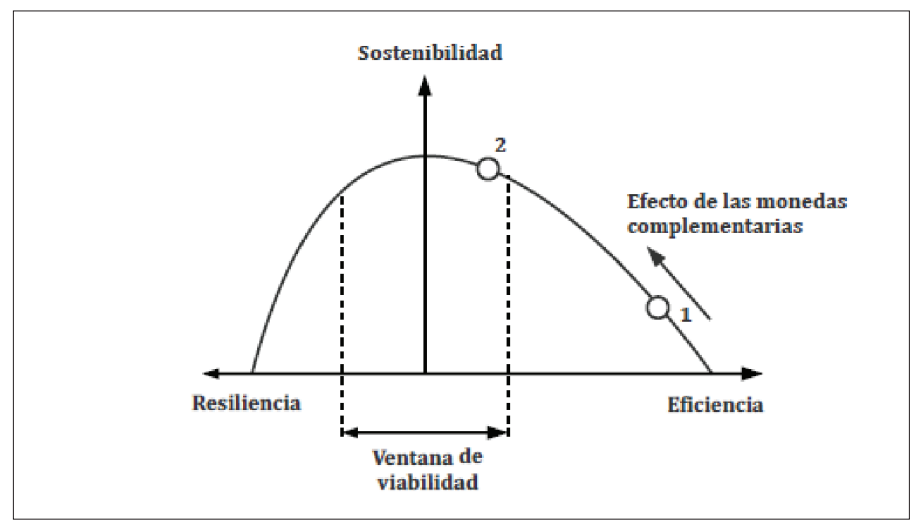

Fuente: Corrons (2015), adaptado de Lietaer y otros (2012).

En el caso de que suceda tras un colapso o crisis, la Figura 9 muestra que el efecto de las monedas complementarias es análogo al de cualquier sistema natural: el sistema recupera su eficiencia progresivamente, sin perder mucha resiliencia, y acercándose cada vez más a su punto óptimo de sostenibilidad (Lietaer y otros, 2008). 
Figura 9: Recuperación del sistema monetario tras un colapso, con la introducción de monedas complementarias.

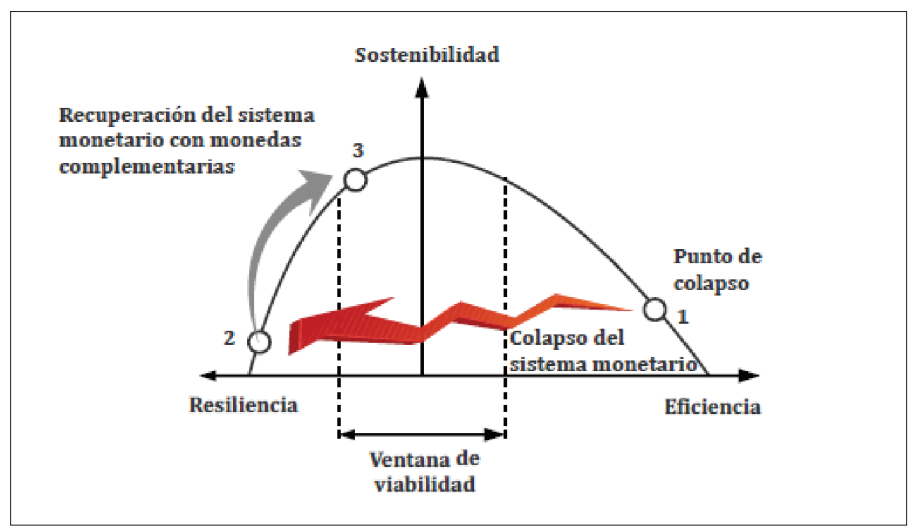

Fuente: Corrons (2015), adaptado de Lietaer y otros (2012).

Se podría decir que el sistema monetario será sostenible y aportará su grano de arena a la sostenibilidad del sistema socio-ecológico en el que vivimos, si y solo si se comporta en una zona en la que convivan tanto la eficiencia como la resiliencia. Ello se traduce en la convivencia de distintas monedas, cada una de las cuales tendrá sus propias funciones y ámbitos de actuación (Lietaer y otros, 2008). Es precisamente este el motivo por el cual en el presente trabajo tratamos a estas monedas como complementarias, puesto que en ningún caso pretenden sustituir o reemplazar a las monedas oficiales o de curso legal, sino al contrario, complementarlas para dar cabida a una serie de transacciones y operaciones que no tendrían lugar.

Un sistema monetario ideal tendería a ser un sistema multi-monetario con una amplia variedad de monedas y de agentes con diferentes ámbitos coexistiendo. Esta nueva configuración iría más allá del dinero como deuda bancaria monopolizado por gigantes financieros y/o Estados, y sería una inteligente estrategia de diversificación, que ampliaría la resiliencia del sistema monetario y, por tanto, la sostenibilidad del mismo (Lietaer y otros, 2008). 


\section{Conclusiones}

A modo de conclusiones, veamos a continuación los resultados más relevantes de este artículo, todos ellos basados en el trabajo de investigación Monedas complementarias en pro de la sostenibilidad y el desarrollo: enfoque panárquico (Corrons, 2015):

El sistema monetario actual, con dinero como deuda bancaria, ha llegado a un punto tal de rigidez y funcionamiento monopolizado que impide tanto su sostenibilidad como la de su entorno.

Las monedas complementarias, en sus distintas tipologías, son herramientas que fomentan la sostenibilidad planetaria y el desarrollo de la especie humana. Aportan valores sociales a las personas y actúan preservando el medio ambiente, todo ello sin perder de vista su importancia en la reactivación económica.

La sostenibilidad del sistema monetario pasa por un compromiso entre la eficiencia y la resiliencia del sistema, debiéndose mantener dentro de la zona de viabilidad correspondiente. El efecto de la introducción de monedas complementarias se traduce en una disminución de la eficiencia y un aumento de la resiliencia, acercándose cada vez más a la zona óptima de sostenibilidad.

Las monedas complementarias, sin ser la panacea, aportan al sistema monetario una serie de valores que no es capaz de transmitir el dinero convencional tal y como lo conocemos a día de hoy. La cooperación, la sostenibilidad, la confianza, todas van implícitamente vinculadas a las monedas complementarias, fomentando una nueva economía, más real y menos especulativa, una ecología más sana y respetuosa, y una sociedad más justa y equitativa. Aun así, hay todavía una barrera difícil de superar en todo ello: la reticencia de la gente a aceptar la transición, a fomentar el cambio, a adaptarse a un entorno dinámico. En este sentido, teorías como la de los sistemas complejos pueden sentar la base de nuevas teorías de cambio que conciencien a las personas y las acompañen en su cambio hacia la construcción de un futuro mejor, hacia un futuro más humano y hacia un planeta Tierra más sostenible. 


\section{Bibliografía}

Brenes, E. (2013): Monedas complementarias y ambiente, Serie Cuides número 10.

CAHN, E. (2000): No more throwaway people: the co-production imperative, Essential Books, Washington.

Collom, E. (2008): «Engagement of the Elderly in Time Banking: The potential for social capital generation in an aging society», Journal of Aging and Social Policy, 20(4).

Corrons, A. (2015): Monedas complementarias en pro de la sosteniblidad y el desarrollo: enfoque panárquico. Trabajo de investigación del Máster Interuniversitario en Cooperación al Desarrollo de la Universitat Jaume I.

Douthwaite, R. (1996): Short circuit: strengthening local economies for security in an uncertain world, Green Books, Totnes.

Ekins, P. (1986): The living economy: a new economics in the making, Routledge, London.

García, R. (2006): Sistemas Complejos. Conceptos, método y fundamentación, Gedisa, México.

Gómez, G. (2008): Making markets. The institutional rise and decline of the Argentine Red de Trueque. Tesis Doctoral, Instituto de Estudios Sociales, La Haya.

GraugaArd, J. (2012): «A tool for building community resilience? A case study of the Lewes Pound», Local Environment, 17(2), pp. 243-260.

Greco, T. (2001): Money understanding and creating alternatives to legal tender, Chelsea Green Publishing Company.

Hopkins, R. (2008): The Transition Handbook: Creating local sustainable communities beyond oil dependency, Finch Publishing, Sydney.

Lietaer, B. y otros (2008): Opciones para gestionar la crisis sistémica de la banca, Documento para la Academia mundial de Artes y Ciencias (WAAS).

LietAer, B. (2005): El futuro del dinero: cómo crear nueva riqueza, trabajo y un mundo más sensato, Errepar-Longseller, Argentina.

Lopezllera, M.L. (2002): Dinero comunitario en México y en el mundo, La Otra Bolsa de Valores, México.

North, P. (2007): Money and liberation, University of Minnesota Press, London.

Pearson, R. (2003): «Argentina's barter networks: new currency for new times», Bulletin of Latin American Research, 22(2), pp. 214-230.

Primavera, H. (2003): «Riqueza, dinero y poder: el efímero milagro argentino de las redes de trueque», en Hintze, S. (2003): Trueque y economía solidaria, 
Universidad Nacional de General Sarmiento, Prometeo Libros, UNDPArgentina, pp. 121-162.

Primavera, H. (2001): Redes de trueque en América Latina: ¿quo vadis?, MS.

Rizzo, P. (2003): L'économie sociale et solidaire face au experimentations monétaire: monnaies socials et monnaies multilaterals, L'Harmattan, Paris.

Ryan-Collins, J. (2011): «Building local resilience: the UK's transition currencies», International Journal of community Currency Research, 15(D), pp. 61-67.

Ryan-Collins, J. y otros (2008): New wealth of time, New Economics Foundation, Londres.

Santana, M.E. (2008): Reinventando el dinero. Experiencias con monedas comunitarias, Tesis Doctoral, Ciesas, México.

Seyfang, G. y N. Longhurst (2013): «Growing green money? Mapping community currencies for sustainable development». Ecological Economics, 86.

Seyfang, G. y N. Longhurst (2012): «Money, Money, Money? A scoping study of Grassroots Complementary currencies for Sustainability», 3S Working Paper 2012-02, University of East Anglia.

Seyfang, G. y A. Smith (2002): The time of our lives: Using time banking for neigbborbood renewal and community capacity building, New Economics Foundation, Londres.

Seyfang, G. (2001b): «Working for the Fenland dollar: an evaluation of local exchange trading schemes (LETS) as an informal employment strategy to tackle social exclusion», Work Employment and Society, 15(3), pp. 581-593.

Seyfang, G. (2001a): «Community currencies: small change for a green economy», Environment and Planning A, 33, pp. 975-996.

STODder, J. (1998): Reciprocal exchange networks. Implications for macroeconomic stability, Lally School of Management and Technology, Rensselaer Polytechnic Institute.

Ulanowicz, R. (2008): A Third Window: Natural Foundations for Life, Oxford University Press, New York.

Ulanowicz, R.y otros (2008): «Quantifying sustainability: efficiency, resilience and the return of information theory», Journal of Ecological Complexity (forthcoming).

Weatherford, J. (1998): La bistoria del dinero. De la piedra arenisca al ciberespacio, Crown Publisher, Santiago de Chile.

Williams, C. y otros (2001): Bridges into work. An evaluation of local exchange trading schemes (LETS), Policy Press, Bristol. 